\title{
Autologous PD-1-targeted Chimeric Switch Receptor-modified T Lymphocytes
}

\author{
National Cancer Institute
}

\section{Source}

National Cancer Institute. Autologous PD-1-targeted Chimeric Switch Receptor-modified T Lymphocytes. NCI Thesaurus. Code C132251.

Autologous human T-lymphocytes that are genetically eng ineered to express a chimeric switch receptor (CSR) composed of the extracellular ligand binding domain of the human inhibitory receptor programmed cell death protein 1 (PD-1; PDCD1) fused to the transmembrane and cytoplasmic co-stimulatory signaling domains of CD28 (PD1CD28; PD-1:CD28 switch receptor), with potential immunomodulating and antineoplastic activities. Upon reintroduction of autologous PD-1-targeted CSR-modified Tlymphocytes into the patient, the switch receptor expressed by the eng ineered T-cells targets and binds to the PD-1 ligands, programmed cell death ligand 1 (PD-L1) and 2 (PD-L2) expressed, on tumor cells. The nature of the PD-1/CD28 switch receptor fusion protein prevents the normal PD1/PD-L1-mediated T-cell suppression and, instead, promotes signaling through the CD28 domain, which results in the stimulation of Tlymphocytes. This induces enhanced toxicity against PD-L1-expressing tumor cells. PD-1 protein, found on activated T-cells, negatively regulates T-cell activity; it plays a key role in immune evasion and prevents tumor cell lysis. Exchanging the transmembrane and intracellular domain of PD-1 with that of CD28 converts PD-L1 into a co-stimulation ligand of primary human CD8+ cytotoxic T-lymphocytes (CT Ls). CD28, is a molecule expressed by $\mathrm{T}$-cells that stimulates increased T-lymphocyte proliferation and activity. 